Journal of Statistical Research

ISSN 0256 - 422X

2019, Vol. 53, No. 1, pp. 27-44

\title{
ASSESSING THE INTER-PATIENT VARIABILITY IN THE AREA UNDER THE CONCENTRATION CURVE
}

\author{
M. IfTAKHAR Alam \\ Institute of Statistical Research and Training, University of Dhaka, Dhaka 1000, Bangladesh \\ Email: iftakhar@isrt.ac.bd
}

\begin{abstract}
SUMMARY
In this paper, we check the accuracy of an approximate variance for the area under the concentration curve (AUC). Based on the model function of a random effects model, an analytic expression for the AUC can be obtained. Then an approximate variance for the AUC can be derived after the linearisation of it using the Taylor series expansion. This variance is used in the construction of confidence interval to check the accuracy of the approximation made. We assume that the data on concentration are collected with the advancement of a dosefinding study. Following the allocation of the lowest dose to a cohort of patients, the concentration of drug in the blood samples is measured at the locally $D$ optimum time points. The successive doses for the cohorts are determined by the popular continual reassessment method. Four PK profiles, along with three plausible dose-response scenarios, are investigated through a simulation study. The numerical findings assure the quality of the approximated variance.
\end{abstract}

Keywords and phrases: Phase I trial; Area under the concentration curve; $D$ optimum sampling times; One-compartment pharmacokinetic model; Continual reassessment method.

AMS Classification: $92 \mathrm{~B} 15$

\section{Introduction}

Clinical trials, commonly classified into four phases, have become an integral part of drug development. Phase I is the first stage of testing in humans and designed to assess safety, tolerability and the pharmacokinetics (PK) of a drug. Phase II is designed to assess how well the drug works (pharmacodynamics, PD) and it also monitors safety in a large group of patients. Pharmacokinetics is generally defined as what the body does to the drug. It reflects the movement of the drug in the body; that is, how the drug enters into the body, how it is distributed throughout the body and how it leaves the body. It involves the study of the processes that affect the plasma concentration of drug in the body at any time after the administration of a dose (Rosenbaum, 2011). After the administration of a dose, the PK mechanism transforms it into plasma concentration and through the systematic circulation of blood, it reaches the site of action and produces the PD response.

(C) Institute of Statistical Research and Training (ISRT), University of Dhaka, Dhaka 1000, Bangladesh. 
The extent of response depends on the concentration at the site of action. We cannot measure concentration at the site of action, but can measure the plasma, which reflects the concentration at the site. Concentration needs to be kept high enough to produce a desirable response, but low enough to avoid toxicity. Even if the same dose is given to a group of individuals, concentration profiles are very likely to be different since it depends on how a body functions. Concentration is inherently more informative than dose because unlike dose, which is only a nominal mass fixed by the clinicians, it gives biological information (Riviere, 2011). Dose-concentration-effect relationship is capable of predicting the effect at any time after administering a dose. It also helps in estimating the dose and dosing interval to achieve the effect of a desired level. Therefore, for the efficient determination of the optimum dose, it is essential to monitor plasma concentration.

A drug's pharmacokinetics are determined by the processes of absorption, distribution, metabolism and excretion (ADME). Also, concentrations at the site of action are determined by the ADME. The objectives in a early phase clinical trial are to quantify the ADME of the drug and also to identify an optimal dose for further studies. Quantifying these PK parameters is often straightforward, as it does not require the administration of an optimal dose (Piantadosi and Liu, 1998). However, obtaining the optimal dose is a difficult task. There are drugs for which the therapeutic range is narrow and as such careful dose escalation is essential since a small increase in dose may lead to a drastic change in the response.

The AUC is a PK parameter that reflects the overall amount of drug in the plasma after the administration of a dose. On the other hand, $\mathrm{C}_{\max }$ is the maximum concentration that can occur any time after the administration of a dose. The unit of AUC is the product of concentration and time. For instance, it could be $\mathrm{mg} / \mathrm{litre} \times \mathrm{hr}$. A non-parametric approach is often used to obtain the AUC. It employs the trapezoidal rule (Gabrielsson and Weiner, 2000) and requires a large number of samples per subject. A larger number of blood samples gives more trapeziums to find an accurate estimate of the area. An alternative could be estimation based on a parametric PK model. For many PK models, it is possible to obtain an analytical form for the AUC by integrating the model function over the sampling region. Use of optimal sampling time points will reduce the number of samples to be collected per subject, and so the parametric approach has an advantage over the non-parametric one. This paper focuses on the parametric approach for finding the AUC, as shown in Section 2.1 .

Since the PD response depends on the concentration at the site of action, it may not be enough to consider only the administered dose in establishing a dose-response relationship. Rather, the resulting concentration from that dose has a vital role in it. There are situations where a small increment in dose may produce a large increase in the AUC (Graham and Workman, 1992). Small AUC values have the capacity to produce no effect and similarly high AUC values have the capacity to lead to toxicity. Since AUC represents the full drug concentration in the body, it can be an excellent measure to assist in drug-dose administration. One possible way could be the formal inclusion of measures like the $\mathrm{AUC}$ or $\mathrm{C}_{\max }$ in the dose-response model. One such method that incorporates AUC as a covariate in the 
dose-response model is that of Piantadosi and Liu (1998). But that method requires many blood samples to be collected since non-parametric approach is used to obtain the AUC. Whitehead et al. (2007) proposed a Bayesian procedure for the simultaneous monitoring of pharmacodynamic and pharmacokinetic responses in phase I trials. Cotterill et al. (2015) developed a method of dose-escalation for a dual-agent treatment which enables formal integration of exposure information into dose-escalation decisions. Ursino et al. (2017) also concluded that use of PK information in the dose allocation process can enrich the knowledge of the dose-toxicity relationship and thus leads to better dose recommendation for subsequent trials. Alam et al. (2017) proposed a method that constrains the AUC for dose finding in seamless phase I/II clinical trials. Takeda et al. (2018) proposed an approach that incorporates into the dose-response model an adjustment based on the latest AUC.

The above discussion reveals that the AUC has important role in the determination of an optimum dose. Unlike dose, the AUC is likely to vary from patient to patient. A generalized method of limited PK sampling has been described in Duffull et al. (1999). The method is designed to enable the accurate estimation of AUC for drugs that are administered intravenously and display two-compartment pharmacokinetics. Gagnon and Leonov (2004) demonstrated a way to optimize the precision of parameter estimates by finding the best number and allocation of sampling times. They found that a reduced number of samples may be taken without significant loss in the precision of parameter estimates. Scheff et al. (2011) developed an algorithm to calculate the AUC with respect to a variable baseline by comparing the AUC of the response curve with the AUC of the baseline while taking into account uncertainty in both measurements. Jawien (2014) proposed a method for the construction of a linear estimator of the AUC in a finite interval that is optimal in the minimax sense. Barnett et al. (2018) proposed a method of producing optimal designs for non-compartmental analysis of pharmacokinetic study.

Bailer (1988) described an way to find the AUC from destructive sampling by using the trapezoidal rule. An expression for variance can be derived easily since the trapezoidal rule is a linear combination of mean concentrations at each sampling time. Yuan (1993) extended this work for obtaining the variance of AUC in animal studies. Many pharmacokinetic studies use log-trapezoidal rule to estimate the AUC. Gagnon and Peterson (1998) obtained a first-order approximation to the variance of AUC for the log-trapezoidal rule. Jaki and Wolfsegger (2012) proposed a non-compartmental estimation approach for the AUC and its variance. In this paper, we check the accuracy of a variance for the AUC that can be obtained from a PK model. The organisation of the paper is as follows. Section 2 presents the methods to be used. It includes the PK model and the dose escalation design that we consider. The settings of simulation and the numerical results are discussed in Section 3 and 4, respectively. Finally, the conclusion appears in Section 5. 


\section{Methods}

Even if the same dose is given to a group of patients, the concentration profiles are very likely to be different. This is due to the differences in biological factors among the patients. As the profiles vary, so do the AUCs, and an obvious issue then is to quantify this variability. In the following section, we employ a non-linear random effects model for the concentration data, since one can assume that each individual has distinct values for the model parameters. As the AUC is derived from model function, it relies on model parameters. The next section describes a way to find the inter-patient variability in the AUC after the administration of a dose to a cohort of patients. Quantifying this variability is important for two particular reasons. Firstly, it will enable us to know the extent of variability from patient to patient, and, secondly, it may guide in dose finding in phase I clinical trials.

Assume that we have some pre-specified doses based on preclinical studies and we want to conduct a phase I trial. We start with allocating the lowest dose to a cohort of patients. Then we record the concentration of drug in the blood samples at the locally $D$-optimum time points, and also, observe the dose-response outcomes. Note that an outcome may be either toxic or non-toxic. After that the PK and dose-response parameters are estimated. Then we select the dose for the second cohort following the continual reassessment method, described in Section 2.2. The dose-response outcomes are observed for the second cohort, and the blood samples are collected at the newly obtained $D$-optimum time points based on the PK parameter estimates at the first stage. We then select dose for the third cohort and the process continues until reaching the maximum number of $m$ cohorts. At each stage of a trial, the dose-response parameter estimates are used to find the next dose and the PK parameter estimates are used to find the locally $D$-optimum time points. At the end of each trial, we construct a confidence interval for the AUC using the final PK parameter estimates. The confidence interval helps to check the accuracy of the approximate variance of the AUC, shown in Section 2.1. The derived variance may help in implementing a PK guided dose escalation study if the approximation is found satisfactory.

\subsection{PK Model and the AUC}

We consider a PK model for the concentration of a drug in the plasma. Often, the mechanistic part of such models is a solution of differential equations representing the distribution of the drug in the body's compartments. The drug is absorbed, distributed and eliminated, and these processes differ among patients. Hence, so-called population PK models are required to account for this variability. As an example, we consider a one-compartment PK

random effects model with bolus input and first-order elimination. The model is defined as

$$
\begin{aligned}
y_{i l} & =f\left(t_{i l} ; \boldsymbol{\theta}_{i}\right)+\epsilon_{i l} \\
& =\frac{x}{V_{i}} \exp \left(-\frac{C l_{i}}{V_{i}} t_{i l}\right)+\epsilon_{i l},
\end{aligned}
$$


where $y_{i l}$ is the concentration of a drug in the blood for the $i$ th individual observed at time $t_{i l}$ and $x$ is the dose received by the individual $(i=1, \ldots, c$ and $l=1, \ldots, n)$. All of the model parameters are assumed random and is represented in an additive form as $\boldsymbol{\theta}_{i}=\boldsymbol{\beta}+\boldsymbol{b}_{i}=\left(V_{i}, C l_{i}\right)^{T}$ with $\boldsymbol{\beta}=(V, C l)^{T}$ and $\boldsymbol{b}_{i}=\left(b_{V_{i}}, b_{C l_{i}}\right)^{T}$. The $\epsilon_{i j}$ denotes the random error, and $n$ is the number of measurements taken on an individual. We assume that $\boldsymbol{\epsilon}_{i} \sim N_{n}\left(\mathbf{0}, \sigma^{2} \boldsymbol{I}_{n}\right)$ and $\boldsymbol{b}_{i} \sim N_{2}(\mathbf{0}, \boldsymbol{\Omega})$, where $\boldsymbol{\Omega}=\operatorname{diag}\left(\sigma_{1}^{2}, \sigma_{2}^{2}\right)$, and also, that $\boldsymbol{b}_{i}$ and $\boldsymbol{\epsilon}_{i}$ are independent.

Our interest is in the efficient estimation of all the population parameters, denoted by $\boldsymbol{\Psi}=\left(\boldsymbol{\beta}^{T}, \boldsymbol{\lambda}^{T}\right)^{T}$, where $\boldsymbol{\beta}^{T}=\left(\beta_{1}, \beta_{2}\right)$ and $\boldsymbol{\lambda}^{T}=\left(\sigma_{1}^{2},, \sigma_{2}^{2}, \sigma^{2}\right)$. We intend to obtain maximum likelihood estimates via non-linear mixed effects model. The Fisher information matrix (FIM) for the $i$ th individual and design $\boldsymbol{\xi}_{i}=\left\{t_{i 1}, \ldots, t_{i n}\right\}$ is given by

$$
\boldsymbol{M}_{i}\left(\boldsymbol{\Psi}, \boldsymbol{\xi}_{i}\right)=\mathrm{E}\left\{-\frac{\partial^{2} l_{i}\left(\boldsymbol{\Psi} \mid \boldsymbol{y}_{i}\right)}{\partial \boldsymbol{\Psi} \partial \boldsymbol{\Psi}^{T}}\right\}
$$

where $l_{i}\left(\boldsymbol{\Psi} \mid \boldsymbol{y}_{i}\right)$ is the log-likelihood function for a given vector of observations $\boldsymbol{y}_{i}=$ $\left(y_{i 1}, \ldots, y_{i n}\right)^{T}$ for individual $i$. We cannot derive an analytic expression for the log-likelihood function, as our model is non-linear in the parameters. Various approximations to the information matrix have been proposed in the statistical literature. A comparison of different methods is presented in Mielke (2012), who concludes that none of the methods is uniformly best.

We approximate the log-likelihood function using a first-order Taylor series expansion of the function $f\left(t_{i j} ; \boldsymbol{\theta}_{i}\right)$ about $\boldsymbol{\phi}_{i}=\left(\boldsymbol{\beta}, \boldsymbol{b}_{i}\right)^{T}$ at $\boldsymbol{\phi}^{0}=\left(\boldsymbol{\beta}^{0}, E\left(\boldsymbol{b}_{i}\right)\right)^{T}=\left(\boldsymbol{\beta}^{0}, \mathbf{0}\right)^{T}$. It can be shown that the population FIM for the design $\boldsymbol{\Xi}=\left\{\boldsymbol{\xi}_{1}, \ldots, \boldsymbol{\xi}_{c}\right\}$ is defined as the sum of the $c$ individual Fisher information matrices in (2.2). That is,

$$
\boldsymbol{M}(\boldsymbol{\Psi}, \boldsymbol{\Xi})=\sum_{i=1}^{c} \boldsymbol{M}_{i}\left(\boldsymbol{\Psi}, \boldsymbol{\xi}_{i}\right)
$$

where $\boldsymbol{M}_{i}\left(\boldsymbol{\Psi}, \boldsymbol{\xi}_{i}\right)$ is the FIM for an individual. Following the model in (2.1), we can write

$$
\boldsymbol{f}\left(\boldsymbol{\xi}_{i} ; \boldsymbol{\theta}_{i}\right)=\left[\begin{array}{c}
\frac{x}{V_{i}} \exp \left(-\frac{C l_{i}}{V_{i}} t_{i 1}\right) \\
\frac{x}{V_{i}} \exp \left(-\frac{C l_{i}}{V_{i}} t_{i 2}\right) \\
\vdots \\
\frac{x}{V_{i}} \exp \left(-\frac{C l_{i}}{V_{i}} t_{i n}\right)
\end{array}\right] .
$$

To linearise the model in (2.1), we expand it using a first-order Taylor series about $\phi_{i}=\left(V, C l, b_{V_{i}}, b_{C l_{i}}\right)^{T}$ at $\phi^{0}=\left(V^{0}, C l^{0}, 0,0\right)^{T}$. Following the expansion, we can obtain an approximated linear mixed effects model of the form

$$
\boldsymbol{y}_{\boldsymbol{i}} \cong \boldsymbol{\mu}_{i}+\boldsymbol{H}_{i} \boldsymbol{\beta}+\boldsymbol{L}_{i} \boldsymbol{b}_{i}+\boldsymbol{\epsilon}_{i}
$$


where $\boldsymbol{\mu}_{i}$ is a vector of constants and

$$
\begin{aligned}
\boldsymbol{H}_{i} & =\left[\begin{array}{cc}
\frac{\partial f\left(t_{i 1} ; \boldsymbol{\theta}_{i}\right)}{\partial V} & \frac{\partial f\left(t_{i 1} ; \boldsymbol{\theta}_{i}\right)}{\partial C l} \\
\vdots & \vdots \\
\frac{\partial f\left(t_{i n} ; \boldsymbol{\theta}_{i}\right)}{\partial V} & \frac{\partial f\left(t_{i n} ; \boldsymbol{\theta}_{i}\right)}{\partial C l}
\end{array}\right]_{\phi^{0}} \\
& =\left[\begin{array}{cc}
\frac{x}{\left(V^{0}\right)^{2}} \exp \left(-\frac{C l^{0}}{V^{0}} t_{i 1}\right)\left(\frac{C l^{0}}{V^{0}} t_{i 1}-1\right) & -\frac{x t_{i 1}}{\left(V^{0}\right)^{2}} \exp \left(-\frac{C l^{0}}{V^{0}} t_{i 1}\right) \\
\vdots & \vdots \\
\frac{x}{\left(V^{0}\right)^{2}} \exp \left(-\frac{C l^{0}}{V^{0}} t_{i n}\right)\left(\frac{C l^{0}}{V^{0}} t_{i n}-1\right) & -\frac{x t_{i n}}{\left(V^{0}\right)^{2}} \exp \left(-\frac{C l^{0}}{V^{0}} t_{i n}\right)
\end{array}\right] .
\end{aligned}
$$

Since $\boldsymbol{\theta}_{i}=\boldsymbol{\beta}+\boldsymbol{b}_{i}$, we have $\boldsymbol{H}_{i}=\boldsymbol{L}_{i}$, where

$$
\boldsymbol{L}_{i}=\left[\begin{array}{cc}
\frac{\partial f\left(t_{i 1} ; \boldsymbol{\theta}_{i}\right)}{\partial b_{V_{i}}} & \frac{\partial f\left(t_{i 1} ; \boldsymbol{\theta}_{i}\right)}{\partial b_{C l_{i}}} \\
\vdots & \vdots \\
\frac{\partial f\left(t_{i n} ; \boldsymbol{\theta}_{i}\right)}{\partial b_{V_{i}}} & \frac{\partial f\left(t_{i n} ; \boldsymbol{\theta}_{i}\right)}{\partial b_{C l_{i}}}
\end{array}\right]_{\phi^{0}} .
$$

Furthermore, $E\left(\boldsymbol{y}_{i}\right) \cong \boldsymbol{E}_{i}=\boldsymbol{\mu}_{i}+\boldsymbol{H}_{i} \boldsymbol{\beta}$ and $\operatorname{Var}\left(\boldsymbol{y}_{i}\right) \cong \boldsymbol{V}_{i}=\boldsymbol{L}_{i} \boldsymbol{\Omega L}_{i}^{T}+\sigma^{2} \boldsymbol{I}_{n}$. Since $\boldsymbol{b}_{i}$ and $\boldsymbol{\epsilon}_{i}$ are assumed to be normal, the log-likelihood function is approximated by

$$
\ell_{i}\left(\boldsymbol{\Psi} \mid \boldsymbol{y}_{i}\right) \cong \log \left[(2 \pi)^{-\frac{n}{2}}\left|\boldsymbol{V}_{\boldsymbol{i}}\right|^{-\frac{1}{2}} \exp \left\{-\frac{1}{2}\left(\boldsymbol{y}_{i}-\boldsymbol{E}_{i}\right)^{T} \boldsymbol{V}_{i}^{-1}\left(\boldsymbol{y}_{i}-\boldsymbol{E}_{i}\right)\right\}\right] .
$$

Then the FIM for the $i$ th individual can be approximated by the block diagonal matrix

$$
\boldsymbol{M}_{i}\left(\boldsymbol{\Psi}, \boldsymbol{\xi}_{i}\right) \cong\left[\begin{array}{cc}
\boldsymbol{A}_{i} & \mathbf{0} \\
\mathbf{0} & \boldsymbol{B}_{i}
\end{array}\right]
$$

where

$$
\begin{aligned}
& \left.\left(\boldsymbol{A}_{i}\right)_{m l}=\left(\frac{\partial \boldsymbol{E}_{i}}{\partial \beta_{m}}\right)^{T} \boldsymbol{V}_{i}^{-1} \frac{\partial \boldsymbol{E}_{i}}{\partial \beta_{l}} \text { for } m, l=1,2 \text { and } \beta_{1}=V, \beta_{2}=C l\right), \\
& \left(\boldsymbol{B}_{i}\right)_{m l}=\frac{1}{2} \operatorname{tr}\left(\frac{\partial \boldsymbol{V}_{i}}{\partial \lambda_{m}} \boldsymbol{V}_{i}^{-1} \frac{\partial \boldsymbol{V}_{i}}{\partial \lambda_{l}} \boldsymbol{V}_{i}^{-1}\right) \text { for } m, l=1,2,3 \text { and } \boldsymbol{\lambda}=\left(\sigma_{1}^{2}, \sigma_{2}^{2}, \sigma^{2}\right)^{T} .
\end{aligned}
$$

Retout et al. (2001) used a first-order Taylor series expansion of the model function $f$ about the expectation of the parameters to linearise the model. Although their linearised model is different from ours in (2.3), we have found that the FIMs in both cases are the same. To derive the FIM in their approach, one needs to assume that $\boldsymbol{V}_{i}$ is independent of $\boldsymbol{\beta}$. But their expression for $\boldsymbol{V}_{i}$ clearly indicated that this would not be the case. However, in our approach, we do not need to make any such assumption. Both FIMs produce the same design, as in the design optimality criteria only a function of the FIM is used, and 
it will not matter whether the two linearised models are exactly the same. In this paper, we use the $D$-optimality criterion, that is, we maximize the determinant of the population FIM to obtain sampling times for measuring the concentration of the drug in the plasma. Computations for the $D$-optimal time points are implemented in PFIM 3.2 (Bazzoli et al., 2010 ), an $R$ package to evaluate and optimize designs in the context of population PK experiments.

Given the dose $x$ to a cohort of patients, let $h\left(x, \boldsymbol{\theta}_{\boldsymbol{i}}\right)$ be the AUC for individual $i$ in that group based on a PK model. Here, $\boldsymbol{\theta}_{\boldsymbol{i}}$ is the vector of random PK parameters introduced earlier and $h$ is a differentiable function of the parameters. Since the parameters are random, individuals in the cohort will have different AUC values. Alam et al. (2017) derived an expression to assess this inter-patient variability. Since a PK model is non-linear in the parameters, the AUC will be too. Therefore, we linearise the function using a first-order Taylor series expansion of $h\left(x, \boldsymbol{\theta}_{\boldsymbol{i}}\right)$ about $\boldsymbol{\theta}_{\boldsymbol{i}}$ at $E\left(\boldsymbol{\theta}_{\boldsymbol{i}}\right)$ to obtain approximate AUC and its variance. The derivation is based on the $\delta$-method (Oehlert, 1992).

The linearisation of the function using a first-order Taylor series expansion of $h\left(x, \boldsymbol{\theta}_{\boldsymbol{i}}\right)$ about $\boldsymbol{\theta}_{\boldsymbol{i}}$ at $E\left(\boldsymbol{\theta}_{\boldsymbol{i}}\right)$ results into

$$
\begin{aligned}
h\left(x, \boldsymbol{\theta}_{\boldsymbol{i}}\right) & \left.\cong h\left(x, \boldsymbol{\theta}_{\boldsymbol{i}}\right)\right|_{E\left(\boldsymbol{\theta}_{\boldsymbol{i}}\right)}+\left.\left(\frac{\partial h\left(x, \boldsymbol{\theta}_{\boldsymbol{i}}\right)}{\partial \boldsymbol{\theta}_{\boldsymbol{i}}}\right)^{T}\right|_{E\left(\boldsymbol{\theta}_{\boldsymbol{i}}\right)}\left(\boldsymbol{\theta}_{\boldsymbol{i}}-E\left(\boldsymbol{\theta}_{\boldsymbol{i}}\right)\right) \\
& =h(x, \boldsymbol{\beta})+\left.\left(\frac{\partial h\left(x, \boldsymbol{\theta}_{\boldsymbol{i}}\right)}{\partial \boldsymbol{\theta}_{\boldsymbol{i}}}\right)^{T}\right|_{E\left(\boldsymbol{\theta}_{\boldsymbol{i}}\right)} \boldsymbol{b}_{\boldsymbol{i}},
\end{aligned}
$$

where $\boldsymbol{\theta}_{i}=\boldsymbol{\beta}+\boldsymbol{b}_{i}$ and $E\left(\boldsymbol{\theta}_{i}\right)=\boldsymbol{\beta}$. Since $\boldsymbol{b}_{i} \sim N_{p}(\mathbf{0}, \boldsymbol{\Omega}), h\left(x, \boldsymbol{\theta}_{\boldsymbol{i}}\right)$ will be normally distributed as well. Therefore, $E\left\{h\left(x, \boldsymbol{\theta}_{\boldsymbol{i}}\right)\right\} \cong h(x, \boldsymbol{\beta})$ and

$$
\begin{aligned}
&\left.\left.\operatorname{Var}\left\{h\left(x, \boldsymbol{\theta}_{\boldsymbol{i}}\right)\right\} \cong\left(\frac{\partial h\left(x, \boldsymbol{\theta}_{\boldsymbol{i}}\right)}{\partial \boldsymbol{\theta}_{\boldsymbol{i}}}\right)^{T}\right|_{E\left(\boldsymbol{\theta}_{\boldsymbol{i}}\right)} \operatorname{Var}\left(\boldsymbol{b}_{\boldsymbol{i}}\right)\left(\frac{\partial h\left(x, \boldsymbol{\theta}_{\boldsymbol{i}}\right)}{\partial \boldsymbol{\theta}_{\boldsymbol{i}}}\right)\right|_{E\left(\boldsymbol{\theta}_{\boldsymbol{i}}\right)} \\
&=\left.\left.\left(\frac{\partial h\left(x, \boldsymbol{\theta}_{\boldsymbol{i}}\right)}{\partial \boldsymbol{\theta}_{\boldsymbol{i}}}\right)^{T}\right|_{E\left(\boldsymbol{\theta}_{\boldsymbol{i}}\right)} \boldsymbol{\Omega}\left(\frac{\partial h\left(x, \boldsymbol{\theta}_{\boldsymbol{i}}\right)}{\partial \boldsymbol{\theta}_{\boldsymbol{i}}}\right)\right|_{E\left(\boldsymbol{\theta}_{\boldsymbol{i}}\right)} .
\end{aligned}
$$

The above is a general expression for the variance and therefore can be applied to any PK model. Now we use it for the model in (2.1). The AUC for an individual $i$ over the range $\left[0, t_{1}\right]$ is defined as

$$
\begin{aligned}
h\left(x, \boldsymbol{\theta}_{\boldsymbol{i}}\right) & =\int_{0}^{t_{1}} f\left(t ; \boldsymbol{\theta}_{\boldsymbol{i}}\right) d t \\
& =\int_{0}^{t_{1}} \frac{x}{V_{i}} \exp \left(-\frac{C l_{i}}{V_{i}} t\right) d t \\
& =\frac{x}{C l_{i}}\left\{1-\exp \left(-\frac{C l_{i}}{V_{i}} t_{1}\right)\right\} .
\end{aligned}
$$


Assuming that $E\left(V_{i}\right)=V$ and $E\left(C l_{i}\right)=C l$, we obtain

$$
E\left\{h\left(x, \boldsymbol{\theta}_{\boldsymbol{i}}\right)\right\} \cong h(x, \boldsymbol{\beta})=\frac{x}{C l}\left\{1-\exp \left(-\frac{C l}{V} t_{1}\right)\right\} .
$$

Also,

$$
\begin{aligned}
& \left.\left.\operatorname{Var}\left\{h\left(x, \boldsymbol{\theta}_{\boldsymbol{i}}\right)\right\} \cong\left(\frac{\partial h\left(x, \boldsymbol{\theta}_{\boldsymbol{i}}\right)}{\partial \boldsymbol{\theta}_{\boldsymbol{i}}}\right)^{T}\right|_{E\left(\boldsymbol{\theta}_{\boldsymbol{i}}\right)} \boldsymbol{\Omega}\left(\frac{\partial h\left(x, \boldsymbol{\theta}_{\boldsymbol{i}}\right)}{\partial \boldsymbol{\theta}_{\boldsymbol{i}}}\right)\right|_{E\left(\boldsymbol{\theta}_{\boldsymbol{i}}\right)}
\end{aligned}
$$

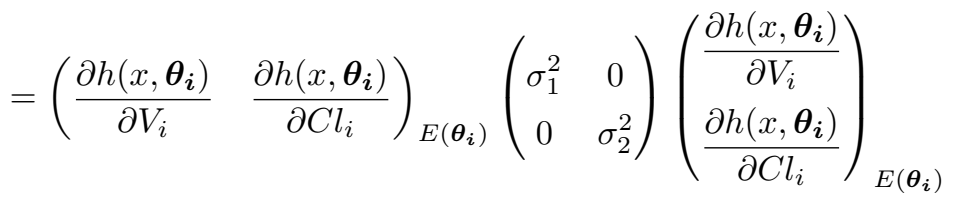

$$
\begin{aligned}
& =\left\{\left.\frac{\partial h\left(x, \boldsymbol{\theta}_{\boldsymbol{i}}\right)}{\partial V_{i}}\right|_{E\left(\boldsymbol{\theta}_{\boldsymbol{i}}\right)}\right\}^{2} \sigma_{1}^{2}+\left\{\left.\frac{\partial h\left(x, \boldsymbol{\theta}_{\boldsymbol{i}}\right)}{\partial C l_{i}}\right|_{E\left(\boldsymbol{\theta}_{\boldsymbol{i}}\right)}\right\}^{2} \sigma_{2}^{2} .
\end{aligned}
$$

Furthermore,

$$
\begin{aligned}
& \left.\frac{\partial h\left(x, \boldsymbol{\theta}_{\boldsymbol{i}}\right)}{\partial V_{i}}\right|_{E\left(\boldsymbol{\theta}_{\boldsymbol{i}}\right)}=-\frac{x t_{1}}{V^{2}} \exp \left(-\frac{C l}{V} t_{1}\right) \\
& \left.\frac{\partial h\left(x, \boldsymbol{\theta}_{\boldsymbol{i}}\right)}{\partial C l_{i}}\right|_{E\left(\boldsymbol{\theta}_{\boldsymbol{i}}\right)}=\frac{x}{C l} \exp \left(-\frac{C l}{V} t_{1}\right)\left(\frac{1}{C l}+\frac{t_{1}}{V}\right)-\frac{x}{C l^{2}} .
\end{aligned}
$$

Hence, we obtain the approximate variance of the AUC as

$$
\begin{aligned}
\operatorname{Var}\left\{h\left(x, \boldsymbol{\theta}_{\boldsymbol{i}}\right)\right\} \cong & \left\{-\frac{x t_{1}}{V^{2}} \exp \left(-\frac{C l}{V} t_{1}\right)\right\}^{2} \sigma_{1}^{2} \\
& +\left\{\frac{x}{C l} \exp \left(-\frac{C l}{V} t_{1}\right)\left(\frac{1}{C l}+\frac{t_{1}}{V}\right)-\frac{x}{C l^{2}}\right\}^{2} \sigma_{2}^{2} .
\end{aligned}
$$

The derived expression can be used to find the inter-patient variability in the AUC based on a PK model after the administration of a dose to a cohort of patients. Also, we have $h\left(x, \boldsymbol{\theta}_{\boldsymbol{i}}\right) \sim N\left[h(x, \boldsymbol{\beta}), \operatorname{Var}\left\{h\left(x, \boldsymbol{\theta}_{\boldsymbol{i}}\right)\right\}\right]$. Therefore, for an individual, we can find an

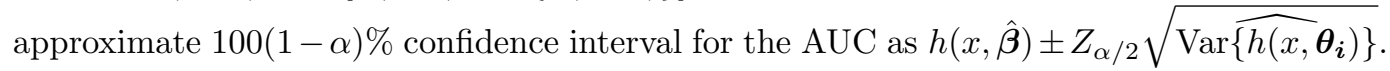
The accuracy of these confidence intervals depends on the quality of the approximated variance. If it is a good approximation of the true variance, the coverage probabilities should be close to the assumed confidence level. Note that the coverage probability refers to the proportion of confidence intervals that include the true AUC.

\subsection{Dose Escalation Design}

Assume that we have $d$ ordered doses $\mathcal{X}=\left\{x^{(1)}, \ldots, x^{(d)}\right\}$ for an experimental drug to find the maximum tolerated dose (MTD). The continual reassessment method (CRM) is a popular model-based procedure to find an MTD (O'Quigley et al., 1990). Assume that we have 
binary response so that 1 means toxic and 0 means a non-toxic outcome. Then the design utilizes the following logistic model to assess the dose-response relationship accurately:

$$
\psi(x, \boldsymbol{\vartheta})=\frac{\exp \left(\vartheta_{1}+\vartheta_{2} x\right)}{1+\exp \left(\vartheta_{1}+\vartheta_{2} x\right)},
$$

where $\boldsymbol{\vartheta}=\left(\vartheta_{1}, \vartheta_{2}\right)$ is the vector of dose-response parameters and $x$ is the dose given to a cohort of patients. The design starts with the lowest dose, and the successive cohorts receive doses from $\mathcal{X}$ following the criterion in (2.6). At the $k$ th stage of a trial, we have the dose and outcome vectors as $\boldsymbol{x}$ and $\boldsymbol{r}$, respectively. Each of these is a $k \times 1$ vector comprising information obtained from $k$ cohorts. Note that there are $c$ patients in a cohort. The likelihood function at the $k$ th stage of a trial is formed as

$$
L_{k}(\boldsymbol{\vartheta} \mid \boldsymbol{x}, \boldsymbol{r}) \propto \prod_{l=1}^{k}\left\{\psi\left(x_{l}, \boldsymbol{\vartheta}\right)\right\}^{r_{l}}\left\{1-\psi\left(x_{l}, \boldsymbol{\vartheta}\right)\right\}^{c-r_{l}} .
$$

The Bayesian approach is engaged to estimate the dose-response parameters $\boldsymbol{\vartheta}$, as the data remains small at the early stages of a phase I trial. The posterior means of the parameters are obtained as

$$
\hat{\vartheta}_{i k}=\frac{\int_{\Theta} \vartheta_{i} g(\boldsymbol{\vartheta}) L_{k}(\boldsymbol{\vartheta} \mid \boldsymbol{x}, \boldsymbol{r}) d \boldsymbol{\vartheta}}{\int_{\Theta} g(\boldsymbol{\vartheta}) L_{k}(\boldsymbol{\vartheta} \mid \boldsymbol{x}, \boldsymbol{r}) d \boldsymbol{\vartheta}}, \quad i=1,2,
$$

where $\Theta$ is the parameter space and $g(\boldsymbol{\vartheta})$ is the prior distribution of the parameters. The uniform prior distribution is used to estimate the parameters. If we assume that $u_{1}<\vartheta_{1}<u_{2}$ and $u_{3}<\vartheta_{2}<u_{4}$, then the restricted parameter space is $\tilde{\Theta}=\left\{\boldsymbol{\vartheta}: u_{1}<\vartheta_{1}<u_{2}, \quad u_{3}<\vartheta_{2}<u_{4}\right\}$. The joint uniform prior distribution then becomes

$$
g(\boldsymbol{\vartheta})=\frac{1}{\left(u_{2}-u_{1}\right)\left(u_{4}-u_{3}\right)}, \quad \boldsymbol{\vartheta} \in \tilde{\Theta} .
$$

The probability of toxicity at the end of stage $k$ is then updated at each available dose as

$$
\hat{\psi}_{i k}=\psi\left(x^{(i)}, \hat{\vartheta}_{k}\right), \quad i=1,2, \ldots, d .
$$

Once the updated estimate of probability of toxicity is available, we choose a dose for the next cohort $k+1$ in the way so that

$$
x_{k+1}=\arg \min _{x \in \mathcal{X}}\left|\psi\left(x, \hat{\vartheta}_{k}\right)-\gamma\right| .
$$

The trial continues until a fixed sample size $m$ is achieved, and the MTD is the dose that would be allocated to patient $m+1$ if $\mathrm{s} /$ he were in the trial. Note that we do not allow the design to skip more than one pre-specified dose level at a time following the suggestion by Goodman et al. (1995). 


\section{Setup to Simulations}

Figure 1 shows three plausible dose-response scenarios, each with the set of doses as $\mathcal{X}=$ $\{1,2, \ldots, 10\}$. The scenarios differ only in terms of shape with the decrease in toxicity if we move from Scenario 1 to Scenario 3. We assume the target toxicity rate $\gamma$ to be 0.33 . The true MTDs in the assumed scenarios are 3,5 and 8, respectively since these are the doses at which the probability of toxicity is close to the target toxicity rate. In contrast, four PK profiles are assumed for each of the scenarios.
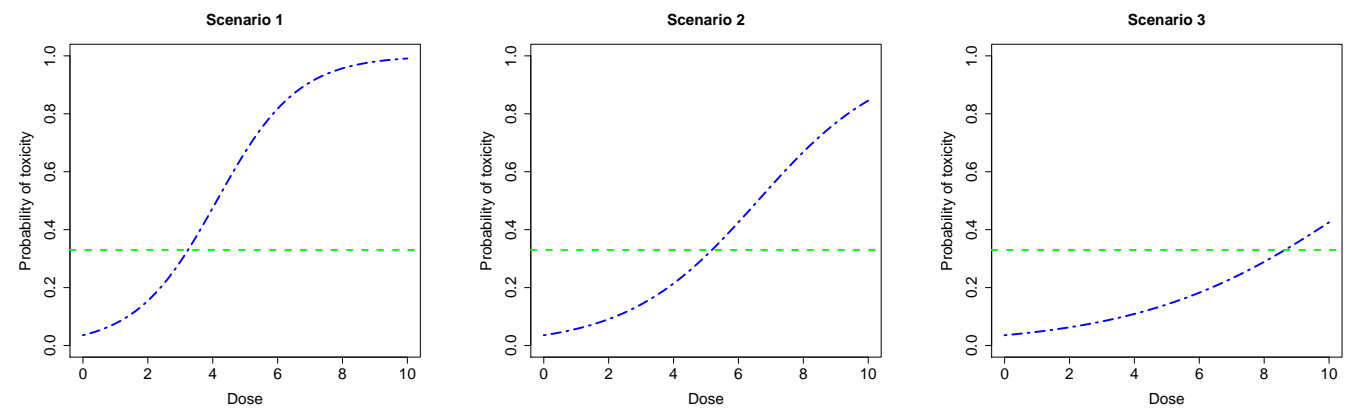

Figure 1: Dose-response scenarios for simulation study. The parameter values in successive scenarios are $\boldsymbol{\vartheta}=(-3.3,0.8), \boldsymbol{\vartheta}=(-3.3,0.5)$ and $\boldsymbol{\vartheta}=(-3.3,0.3)$, respectively. The dotted horizontal line indicates the target toxicity rate $\gamma$.

The mean PK parameter values $V=5 \mathrm{~L}, C l=0.5 \mathrm{~L} / \mathrm{hr}$ and error variance $\sigma^{2}=0.0001$ remain the same across the profiles. But in each profile the variance components $\sigma_{1}^{2}$ and $\sigma_{2}^{2}$ are chosen in a way so that the percentage of coefficient of variation $(\mathrm{CV})$ in the mean parameters is either $15,20,25$ or 30 . For instance, with $15 \% \mathrm{CV}$, the variance components are $\sigma_{1}^{2}=0.563$ and $\sigma_{2}^{2}=0.006$. Small error variance is chosen, as we have found generated concentrations to be small.

We consider a bivariate uniform prior distribution for the dose-response parameters $\vartheta$. A single parameter space $\tilde{\Theta}$ is chosen for the scenarios. As it has been found to allow a wide range of dose-response scenarios, we consider $\tilde{\Theta}=\left\{\boldsymbol{\vartheta}:-4.3<\vartheta_{1}<-2.3, \quad 0<\vartheta_{2}<1\right\}$. For the first cohort, initial mean PK parameters are chosen to be three standard deviations above the true values and the variance components and error variance are assumed to be onethird of the true values to find the $D$-optimal time points. Three time points are considered per patient, that is, $n=3$ in (2.1), as it has been revealed that attempt for more time points leads to the occurrence of some similar points. Note that the samples are collected in the period $(0,30)$ hours, that is, $t_{1}=30$. In Figure 2, we compare the determinant of FIM of a 3-point design with that of a 2-point design, a 4-point design with that of a 3-point design and so on. The relative efficiency is obtained as the ratio of the two determinants. Three time points are chosen to collect blood samples as not appreciable gain is achieved in the determinant of the Fisher information matrix if we move from a 3-point design to 


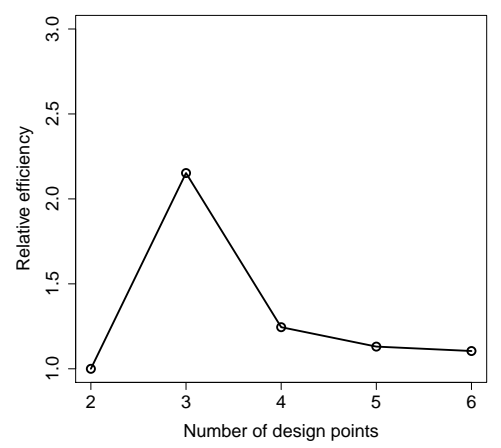

Figure 2: Rationale for setting the number of design points in the one-compartment PK model with bolus input and first-order elimination. The locally $D$-optimum design points are obtained using the initial prior values for the PK parameters, assuming that the lowest dose is given to a cohort of three patients.

a 4-point design. The dose-response outcomes for a cohort are generated from a binomial distribution. The mechanistic part in (2.1) is generated first to add with random errors to yield concentrations for a cohort at the $D$-optimum time points.

A trial starts with the lowest dose of $1 \mathrm{mg} / \mathrm{kg}$-body weight assigned to a cohort of $c=3$ patients. Then the concentration of the drug in the blood samples is measured at the locally $D$-optimum time points. The time points are computed using PFIM (Bazzoli et al., 2010) for the initial prior values of the PK parameters. After receiving outcomes from the first cohort, the dose-response parameters are estimated, as described in Section 2.2. The maximum likelihood estimates of the PK parameters are obtained through the $R$ package nlme (Pinheiro and Bates, 2000). A dose selected through the CRM is then applied to a new cohort, and the blood samples are collected at the $D$-optimum time points using the available parameter estimates of the PK model. After receiving the outcomes from the second cohort, both the dose-response and PK parameters are estimated, and a dose is chosen for the third cohort. The process continues until the trial reaches the maximum number of available cohorts $m=15$.

The MTD at the end of a trial is determined as the dose that would be allocated to cohort 16 if that were in the trial. Based on the PK parameter estimates obtained at the end of a trial, we construct a $95 \%$ confidence interval for the AUC, and check whether AUC value for a random individual belongs to that interval. That is, the possibility of LCL $<h\left(x, \boldsymbol{\theta}_{\boldsymbol{i}}\right)<\mathrm{UCL}$ is checked, where LCL and UCL are the lower and upper confidence limits for the AUC, as explained in Section 2.1. Each case is investigated through 1000 simulated trials, and a self written code in $R$ is used to produce the results. 


\section{Simulation Findings}

The coverage probabilities obtained from simulation study are reported in Table 1 . It is seen that when CV of the PK parameters is $15 \%$, the coverage probability remains 0.93 in all the scenarios. As CV increases, the coverage probability decreases for the scenarios. Table 2 presents bias, standard deviation and average standard error of the estimates of model parameters. Generally speaking, all these measures increase for the parameters as CV increases. To investigate the distribution of the estimates, we present box plots for Scenario 2 in Figure 3. As reflected in it, the variability in PK parameter estimates increases with

Table 1: Coverage probabilities obtained after assuming different coefficient of variation for the mean PK parameters.

\begin{tabular}{ccccc}
\hline & \multicolumn{4}{c}{ Coefficient of Variation \% } \\
\cline { 2 - 5 } Scenario & 15 & 20 & 25 & 30 \\
\hline 1 & 0.93 & 0.92 & 0.92 & 0.91 \\
2 & 0.93 & 0.93 & 0.92 & 0.91 \\
3 & 0.93 & 0.92 & 0.92 & 0.90 \\
\hline
\end{tabular}

$\mathrm{CV}$ in Scenario 2. This finding is in line with our finding from Table 2. Figures for other dose-response scenarios are not presented here, as they have found to follow similar pattern. Confidence intervals are therefore likely to be less accurate to include the true AUC with the increase in $\mathrm{CV}$.

Also, the AUC for a random individual will tend to vary highly with the increase in $\mathrm{CV}$. All these will lead to the decrease in coverage probability. If we increase CV from $15 \%$ to $30 \%$, that is double it, then the coverage probability decreases by a maximum of $3 \%$. Similarly, if we compare between $20 \%$ and $30 \%$, then coverage probability is found to decrease by a maximum of $2 \%$. These figures give some insight of what could happen if we keep increasing CV. Here we have constructed $95 \%$ confidence intervals for each case. As the coverage probabilities are fairly close to 0.95 , the approximate confidence intervals are able to give boundaries for the AUC accurately in majority of the cases. This in turn means that the approximated variance, described in Section 2.1, can capture the inter-patient variability in the AUC precisely.

The main purpose of this article is to check the accuracy of the approximated variance of the AUC. As the CRM has been used to allocate doses, we would like to summarize the results for dose allocation and MTD selection in Table 3. The true MTD in Scenario 1 is 3, which is selected in around $73 \%$ of the trials. Dose 4 is selected in around $25 \%$ of the trials as the MTD. Not many trials recommend dose 5 as the MTD. Also, dose 6 and above are not recommended by any of the trials. Most of the cohorts in the trials are treated around the true MTD, as reflected by the percentages in parentheses.

In Scenario 2, the true MTD 5 is selected in around $53 \%$ of the trials. Dose 6 is selected in around $26 \%$ of the trials, while dose 4 is selected in around $16 \%$ of the trials. Dose 4 and 
Table 2: Bias, standard deviation (SD) and average standard error (SE) of the PK model parameter estimates obtained from simulation.

\begin{tabular}{|c|c|c|c|c|c|c|c|}
\hline \multirow[t]{2}{*}{ Scenario } & \multirow[t]{2}{*}{$\% \mathrm{CV}$} & & \multicolumn{5}{|c|}{ Parameter } \\
\hline & & & $V$ & $\mathrm{Cl}$ & $\sigma_{1}^{2}$ & $\sigma_{2}^{2}$ & $\sigma^{2}$ \\
\hline \multirow[t]{12}{*}{1} & 15 & Bias & -0.0101 & -0.0011 & -0.0219 & -0.0002 & $9.31 \times 10^{-7}$ \\
\hline & & $\mathrm{SD}$ & 0.1095 & 0.0115 & 0.1168 & 0.0012 & $2.19 \times 10^{-5}$ \\
\hline & & SE & 0.1122 & 0.0113 & 0.1196 & 0.0012 & $2.17 \times 10^{-5}$ \\
\hline & 20 & Bias & -0.0088 & $5.54 \times 10^{-5}$ & -0.0234 & -0.0003 & $1.24 \times 10^{-7}$ \\
\hline & & $\mathrm{SD}$ & 0.1454 & 0.0156 & 0.2156 & 0.0022 & $2.10 \times 10^{-5}$ \\
\hline & & $\mathrm{SE}$ & 0.1447 & 0.0148 & 0.1991 & 0.0021 & $2.40 \times 10^{-5}$ \\
\hline & 25 & Bias & -0.0242 & -0.0011 & -0.0505 & -0.0006 & $1.64 \times 10^{-6}$ \\
\hline & & $\mathrm{SD}$ & 0.1918 & 0.0190 & 0.3179 & 0.0033 & $2.13 \times 10^{-5}$ \\
\hline & & SE & 0.1844 & 0.0187 & 0.3226 & 0.0033 & $2.22 \times 10^{-5}$ \\
\hline & 30 & Bias & -0.0242 & -0.0011 & -0.7379 & -0.0075 & $1.63 \times 10^{-6}$ \\
\hline & & $\mathrm{SD}$ & 0.1918 & 0.0190 & 0.3179 & 0.0033 & $2.13 \times 10^{-5}$ \\
\hline & & $\mathrm{SE}$ & 0.2254 & 0.0220 & 0.4831 & 0.0046 & $2.17 \times 10^{-5}$ \\
\hline \multirow[t]{12}{*}{2} & 15 & Bias & -0.0007 & -0.0008 & -0.0198 & -0.0001 & $1.12 \times 10^{-7}$ \\
\hline & & $\mathrm{SD}$ & 0.1093 & 0.0114 & 0.1146 & 0.0012 & $2.11 \times 10^{-5}$ \\
\hline & & SE & 0.1109 & 0.0112 & 0.1165 & 0.0012 & $2.04 \times 10^{-5}$ \\
\hline & 20 & Bias & 0.0008 & -0.0008 & -0.0362 & -0.0003 & $4.29 \times 10^{-7}$ \\
\hline & & $\mathrm{SD}$ & 0.1470 & 0.0152 & 0.1979 & 0.0021 & $2.12 \times 10^{-5}$ \\
\hline & & $\mathrm{SE}$ & 0.1496 & 0.0148 & 0.2115 & 0.0021 & $2.10 \times 10^{-5}$ \\
\hline & 25 & Bias & -0.0103 & -0.0015 & -0.0404 & -0.0005 & $-8.07 \times 10^{-8}$ \\
\hline & & $\mathrm{SD}$ & 0.1767 & 0.0188 & 0.3297 & 0.0036 & $2.07 \times 10^{-5}$ \\
\hline & & $\mathrm{SE}$ & 0.1813 & 0.0185 & 0.3116 & 0.0033 & $2.10 \times 10^{-5}$ \\
\hline & 30 & Bias & -0.0175 & -0.0006 & -0.0979 & -0.0006 & $1.61 \times 10^{-6}$ \\
\hline & & SD & 0.2404 & 0.0235 & 0.4714 & 0.0053 & $2.66 \times 10^{-5}$ \\
\hline & & $\mathrm{SE}$ & 0.2196 & 0.0221 & 0.4614 & 0.0046 & $2.41 \times 10^{-5}$ \\
\hline \multirow[t]{12}{*}{3} & 15 & Bias & -0.0063 & $-7.63 \times 10^{-5}$ & -0.0199 & $-8.25 \times 10^{-5}$ & $1.26 \times 10^{-6}$ \\
\hline & & $\mathrm{SD}$ & 0.1101 & 0.0115 & 0.1185 & 0.0011 & $2.10 \times 10^{-5}$ \\
\hline & & $\mathrm{SE}$ & 0.1093 & 0.0111 & 0.1135 & 0.0012 & $2.11 \times 10^{-5}$ \\
\hline & 20 & Bias & 0.0052 & -0.0005 & -0.0132 & -0.0002 & $9.71 \times 10^{-7}$ \\
\hline & & $\mathrm{SD}$ & 0.1458 & 0.0150 & 0.2170 & 0.0021 & $2.12 \times 10^{-5}$ \\
\hline & & $\mathrm{SE}$ & 0.1472 & 0.0148 & 0.2052 & 0.0021 & $2.15 \times 10^{-5}$ \\
\hline & 25 & Bias & 0.0002 & 0.0001 & -0.0459 & -0.0005 & $-7.58 \times 10^{-7}$ \\
\hline & & $\mathrm{SD}$ & 0.1902 & 0.0188 & 0.3149 & 0.0031 & $2.18 \times 10^{-5}$ \\
\hline & & SE & 0.1828 & 0.0184 & 0.3161 & 0.0032 & $2.09 \times 10^{-5}$ \\
\hline & 30 & Bias & -0.0034 & 0.0012 & -0.0717 & -0.0007 & $-1.82 \times 10^{-7}$ \\
\hline & & $\mathrm{SD}$ & 0.2223 & 0.0228 & 0.4702 & 0.0048 & $2.16 \times 10^{-5}$ \\
\hline & & $\mathrm{SE}$ & 0.2256 & 0.0228 & 0.4788 & 0.0049 & $2.20 \times 10^{-5}$ \\
\hline
\end{tabular}




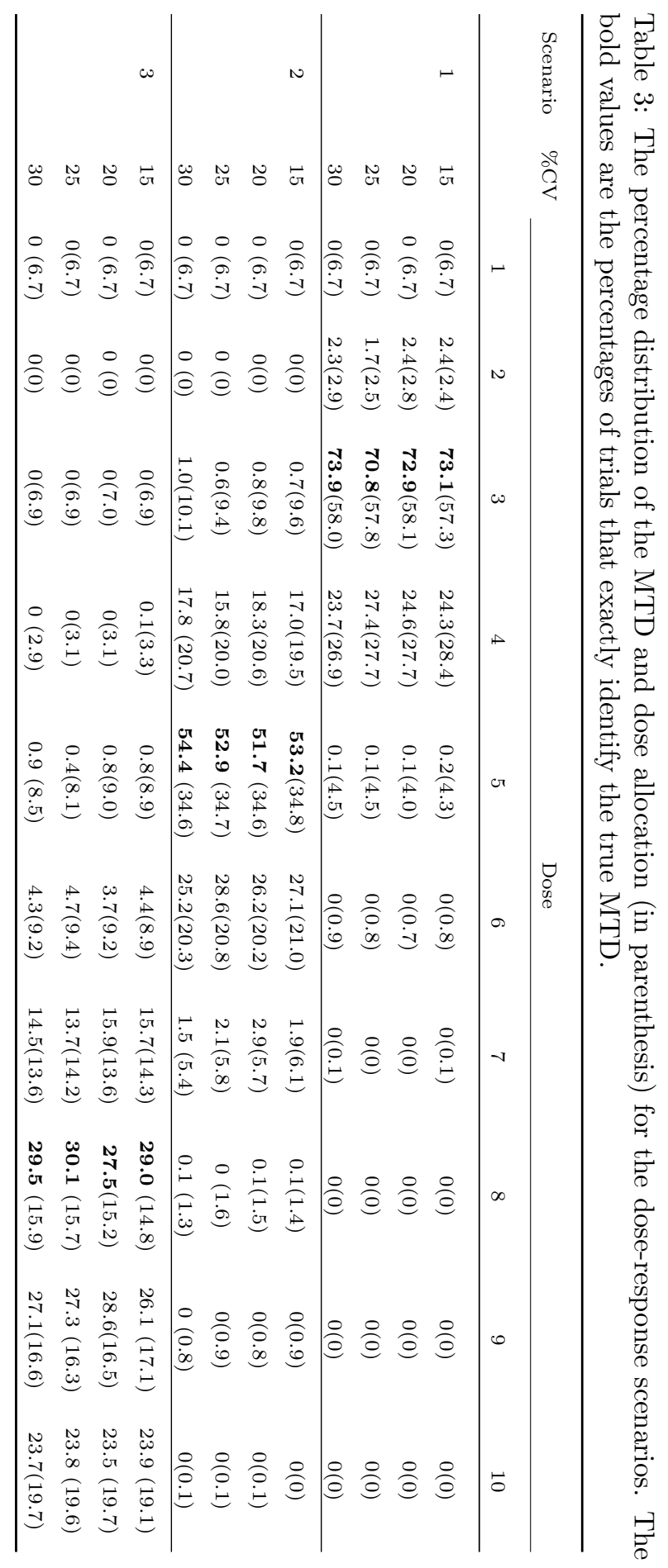



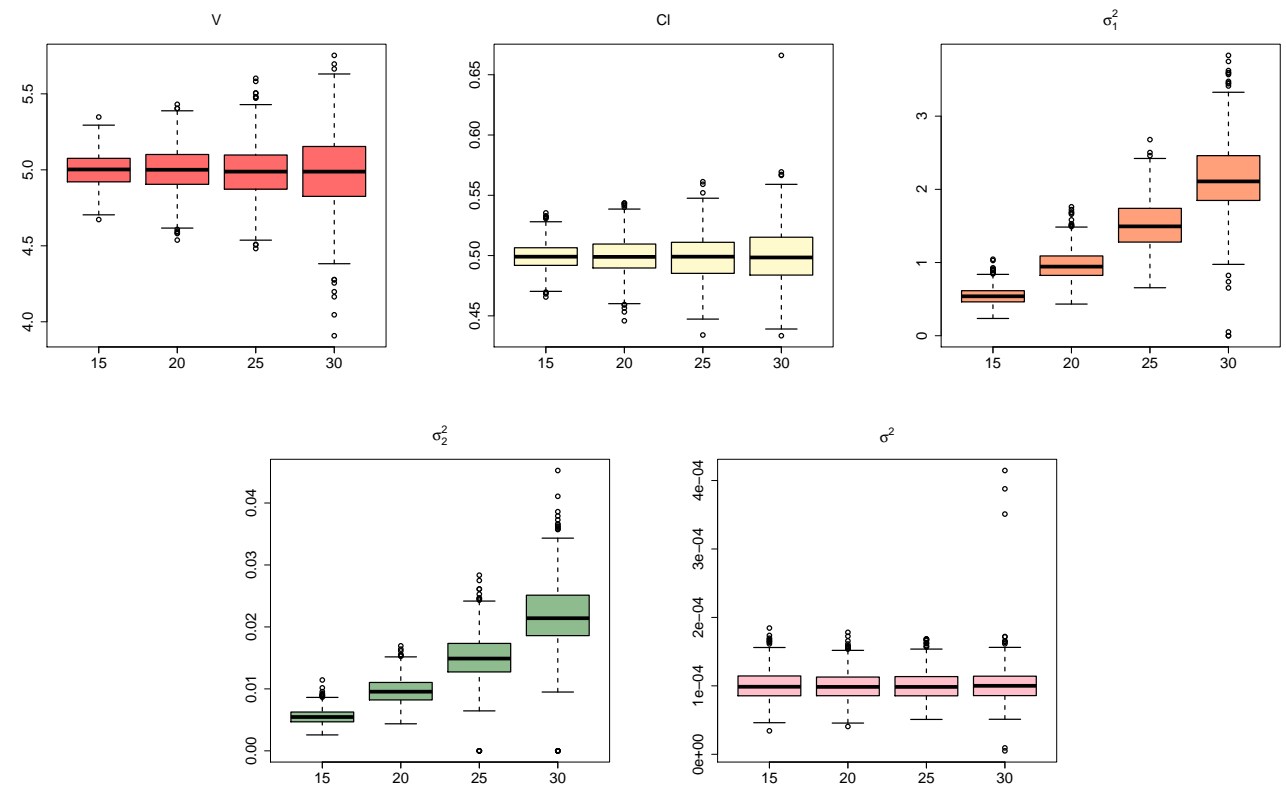

Figure 3: Boxplots of the PK parameter estimates obtained for Scenario 2 in the simulation study. For each parameter, the successive boxes are for 15\%,20\%, 25\% and $30 \%$ of CV, respectively.

6 have the probabilities of toxicity as 0.21 and 0.42 , respectively. Since the latter toxicity is more close to the target toxicity rate 0.33 , dose 6 is selected more often. The cohorts in trials are mostly treated with the doses around the true MTD. The true MTD in Scenario 3 is 8 , and it lies at the upper end of the dose region. It is selected in around $29 \%$ of the trials. Since dose 9 has the probability of toxicity as 0.35 , closer to the target, it is recommended in a good percentage of the trials as well. None of the trials recommend a dose which has a probability of toxicity much lower than the target. Also, majority of the cohorts in this scenario are treated with the doses around 8 .

The property that the CRM dose not expose many patients to either sub-therapeutic or toxic doses is quite evident from the numerical results for the scenarios. As the PK data are used to establish the model parameters and is not used in dose escalation, not appreciable differences are seen in the results across the CVs for a scenario. The seemingly differences are only due to the random variations in the trials. 


\section{Conclusion}

We have investigated the accuracy of an approximated variance for the AUC through a simulation study. Blood samples are collected at the locally $D$-optimal time points to obtain precise estimates of the parameters. To set the number of design points for any working PK model, we suggest to check the relative efficiencies of the designs as we discussed in the paper. As the estimates become more stable towards the end of a trial, the final estimates are used to construct $95 \%$ confidence intervals. The coverage probabilities are found to be close to 0.95 . The more we allow the variability in the mean PK parameters, the more away is the coverage probability from the confidence level. Other confidence levels can be checked as well, but similar results are expected. Similarly, any other dose-response scenarios can be investigated and the results are expected to be in line with the findings here, as we have noticed very little differences in the coverage probabilities across the scenarios.

The expression derived for variance in (2.5) is general and can be applied to any other PK model. Table 1 assures the accuracy of the approximation made. Although the paper limits to the AUC only, a general variance is also possible for the other PK measure $\mathrm{C}_{\max }$ and consequently its accuracy can be checked. Alam et al. (2017) proposed designs for seamless phase I/II clinical trials taking into account such inter-patient variability. One can think of considering the confidence intervals for the AUC or $\mathrm{C}_{\max }$ while allocating doses to the cohorts in a trial. Such consideration will lead to a careful dose escalation design and is particularly important when heterogeneity in ADME is likely to be present in the patient population.

Although the CRM has been used for dose escalation, any other design can be used instead. Also, other dose-response and PK models can be used to apply the presented methodology. As indicated earlier, PK data are not employed in dose escalation. However, the PK parameter estimates obtained at each stage of a trial are used to find the locally $D$ optimum time points. Since the main purpose was to judge the quality of the approximated variance, we refrained ourselves from incorporating AUC in dose escalation. To conclude, we have shown how the inter-patient variability in the AUC, after the assignment of a dose to a cohort of patients, can be assessed from a PK model, and this is when a small number of blood samples are collected from a patient.

\section{Acknowledgements}

The author would like to thank the reviewers for very constructive comments, which have led to an improved version of the paper. Also, special thanks to the editor for helpful suggestions.

\section{References}

Alam, M. I., Bogacka, B., and Coad, D. S. (2017), "Pharmacokinetically guided optimum adaptive dose selection in early phase clinical trials," Computational Statistics and Data 
Analysis, 111, 183-202.

Bailer, A. J. (1988), "Testing for the equality of area under the curves when using destructive measurement techniques," Journal of Pharmacokinetics and Biopharmaceutics, 16, 303309.

Barnett, H. Y., Geys, H., Jacobs, T., and Jaki, T. (2018), "Optimal designs for noncompartmental analysis of pharmacokinetic studies," Statistics in Biopharmaceutical Research, 10, 255-263.

Bazzoli, C., Nguyen, T. T., Dubois, A., Retout, S. Comets, E., and Mentré, F. (2010), "PFIM 3.2 User Guide," Université Paris Diderot and INSERM.

Cotterill, A., Lorand, D., Wang, J., and Jaki, T. (2015), "A practical design for a dual-agent dose-escalation trial that incorporates pharmacokinetic data," Statistics in Medicine, 34, 2138-2164.

Duffull, S., Begg, E., and Deely, J. (1999), "Development of a general method of limited sampling for the determination of AUC for a drug that displays two-compartment pharmacokinetics," European Journal of Clinical Pharmacology, 55, 213-219.

Gabrielsson, J. and Weiner, D. (2000), Pharmacokinetic/Pharmacodynamic Data Analysis: Concepts and Applications, Routledge, London.

Gagnon, R. and Leonov, S. (2004), "Optimal population designs for PK models with serial sampling," Journal of Biopharmaceutical Statistics, 15, 143-163.

Gagnon, R. C. and Peterson, J. J. (1998), "Estimation of confidence intervals for area under the curve from destructively obtained pharmacokinetic data," Journal of Pharmacokinetics and Biopharmaceutics, 26, 87-102.

Goodman, S. N., Zahurak, M. L., and Piantadosi, S. (1995), "Some practical improvements in the continual reassessment method for phase I studies," Statistics in Medicine, 14, $1149-1161$.

Graham, M. A. and Workman, P. (1992), "The impact of pharmacokinetically guided dose escalation strategies in phase I clinical trials: Critical evaluation and recommendations for future studies," Annals of Oncology, 3, 339-347.

Jaki, T. and Wolfsegger, M. J. (2012), "Non-compartmental estimation of pharmacokinetic parameters for flexible sampling designs," Statistics in Medicine, 31, 1059-1073.

Jawień, W. (2014), "Searching for an optimal AUC estimation method: a never-ending task?" Journal of Pharmacokinetics and Pharmacodynamics, 41, 655-673.

Mielke, T. (2012), "Approximation of the Fisher Information and Design in Nonlinear Mixed Effects Models," Ph.D. thesis, Otto-von-Guericke University Magdeburg. 
Oehlert, G. W. (1992), "A note on the delta method," The American Statistician, 46, 27-29.

O'Quigley, J., Pepe, M., and Fisher, L. (1990), "Continual reassessment method: A practical design for phase I clinical trials in cancer," Biometrics, 46, 33-48.

Piantadosi, S. and Liu, G. (1998), "Improved designs for dose escalation studies using pharmacokinetic measurements," Statistics in Medicine, 15, 1605-1618.

Pinheiro, J. C. and Bates, D. M. (2000), Mixed-Effects Models in S and S-PLUS, Springer, New York.

Retout, S., Duffull, S., and Mentré, F. (2001), "Development and implementation of the population Fisher information matrix for the evaluation of population pharmacokinetic designs," Computer Methods and Programs in Biomedicine, 65, 141-151.

Riviere, J. E. (2011), Comparative Pharmacokinetics: Principles, Techniques and Applications, 2nd Edition, Wiley-Blackwell, New York.

Rosenbaum, S. E. (2011), Basic Pharmacokinetics and Pharmacodynamics: An Integrated Textbook and Computer Simulations, Wiley, New York.

Scheff, J. D., Almon, R. R., DuBois, D. C., Jusko, W. J., and Androulakis, I. P. (2011), "Assessment of pharmacologic area under the curve when baselines are variable," Pharmaceutical Research, 28, 1081-1089.

Takeda, K., Komatsu, K., and Morita, S. (2018), "Bayesian dose-finding phase I trial design incorporating pharmacokinetic assessment in the field of oncology," Pharmaceutical Statistics, 17, 725-733.

Ursino, M., Zohar, S., Lentz, F., Alberti, C., Friede, T., Stallard, N., and Comets, E. (2017), "Dose-finding methods for phase I clinical trials using pharmacokinetics in small populations," Biometrical Journal, 59, 804-825.

Whitehead, J., Zhou, Y., Hampson, L., Ledent, E., and Pereira, A. (2007), "A Bayesian approach for dose-escalation in a phase I clinical trial incorporating pharmacodynamic endpoints," Journal of Biopharmaceutical Statistics, 17, 1117-1129.

Yuan, J. (1993), "Estimation of variance for AUC in animal studies," Journal of Pharmaceutical Sciences, 82, 761-763.

Received: May 2, 2019

Accepted: June 9, 2019 